ORIGINAL ARTICLE

\title{
Meningitis is a common cause of convulsive status epilepticus with fever
}

\author{
R F M Chin, B G R Neville, R C Scott
}

Arch Dis Child 2005;90:66-69. doi: 10.1136/adc.2003.038844

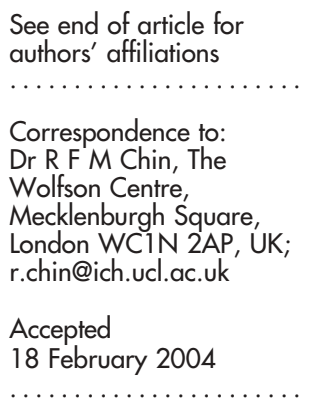

Aims: In children with convulsive status epilepticus (CSE) with fever, to determine the likelihood of acute bacterial meningitis (ABM), the proportion that are treated with antibiotics, and the proportion that have diagnostic CSF sampling.

Methods: Patients with an incident episode of CSE with fever were identified as part of an ongoing prospective population based study of CSE in childhood.

Results: There were 49 incident cases of CSE in the first six months. Ascertainment was $96 \%$. Twenty four had CSE with fever, 16 had early parenteral antibiotics, nine had diagnostic CSF sampling, and four had $A B M$. The population risk of $A B M$ in CSE with fever was significantly higher than that of short seizures with fever (17\% v $1.2 \%)$.

Conclusions: The classical symptoms and signs of ABM may be absent in CSE with fever. A high index of suspicion for $A B M$ in the child with CSE with fever is paramount. The most appropriate management is suggested to be early parenteral antibiotics and a lumbar puncture when there are no contraindications.
S eizures with fever are the most common type of seizures in childhood. ${ }^{1}$ It is essential that associated central nervous system (CNS) infections with their consequent mortality and morbidity are not overlooked. Two recently published reviews reported that only $0.4-1.2 \%$ of children who have a seizure with fever will have unexpected acute bacterial meningitis $(\mathrm{ABM}) .^{2{ }^{3}}$ Thus, it has been suggested that investigation for $\mathrm{ABM}$ is not required in this group, unless other evidence of meningitis is present. A recent guideline on management of children with a seizure suggests that prolonged seizures with fever, at least 15 minutes duration, are more likely to be associated with $\mathrm{ABM}$ than those with short seizures with fever. ${ }^{4}$ This recommendation was derived from hospital based studies, ${ }^{5-7}$ the population risk of $\mathrm{ABM}$ in a child with a prolonged seizure and fever remains unknown.

The aim of this study is to determine the likelihood of ABM in children with convulsive status epilepticus (CSE) associated with fever in the general population, what proportion of children with CSE associated with fever are being treated with antibiotics, and what proportion will have diagnostic cerebrospinal fluid (CSF) sampling.

\section{METHODS}

The patients in this study were identified as part of an ongoing study of CSE in childhood, the North London convulsive STatus EPilepticus in childhood Surveillance Study (NLSTEPSS).

NLSTEPSS is an ongoing prospective population based study that seeks to determine the incidence, aetiology, treatment, and mortality of convulsive status epilepticus (CSE) in North London. Through a collaborative research network of paediatricians from appropriate hospitals in North London, children aged between 29 days and 15 years with CSE (a seizure or series of seizures without recovery of consciousness between seizures, lasting at least 30 minutes) are notified to NLSTEPSS through a two source notification system. Patients are notified prospectively through a telephone hotline available 24 hours per day, and/or through an active regional surveillance system. In the former, trainee paediatricians, nurses, and ward administrators are asked to notify cases of CSE by telephone. In the surveillance system, paediatricians are sent monthly reporting forms with which to notify cases of CSE or to confirm that no cases were identified. After a case is notified, details about the patient and the CSE are obtained from the local paediatric team within two weeks of notification through: (1) anonymised copies of clerking proformas; (2) interview of local paediatricians and or nurses; and (3) anonymised copies of A\&E notes. Patient details requested included unique identifiers such as initials, gender, date of birth, and postal code of residence in order to match cases notified by either or both sources. Details requested about the event included seizure duration, description and treatment, past medical history, investigations and their results, and whether the child survived or not. All patient data were anonymised and entered into an Access database.

Two source capture-recapture analysis, a recognised method for quantification of ascertainment, was applied to assess the completeness of ascertainment of case. ${ }^{8}$ The method of calculation of ascertainment corrected number of cases by two source capture-recapture analysis has been previously described. ${ }^{10}$

During the initial six month period of the study, the NLSTEPSS database was searched for children who had no previous episodes of CSE (incident episodes) and had a fever. The details of their CSE as outlined above, were noted.

NLSTEPSS was approved by the London Multi-Centre Research Ethics Committee.

\section{RESULTS}

In the initial six month period, there were 49 incident cases of CSE notified to NLSTEPSS. Of these, 44 were notified by the telephone hotline, 29 by the active surveillance system, and 25 by both sources. Capture-recapture analysis indicated that $96 \%$ (95\% CI $88-106 \%)$ of incident cases of CSE were ascertained. Of the 49 incident cases, 24 children (49\%) had CSE with fever (above $38^{\circ} \mathrm{C}$ on presentation). The median age

Abbreviations: $A B M$, acute bacterial meningitis; $L P$, lumbar puncture; CNS, central nervous system; CSE, convulsive status epilepticus; CSF, cerebrospinal fluid 
of the children with CSE with fever was 1.3 years (range 2 months to 11.5 years). On presentation, $16(67 \%)$ had parenteral antibiotics for ABM. Diagnostic cerebrospinal fluid (CSF) sampling was conducted in nine $(30 \%)$, eight through lumbar puncture (LP) and one by tapping of a ventriculoperitoneal shunt. Two children had postmortem CSF sampling.

Four (17\%, 95\% CI 15-18\%) children, aged 3 months, 9.5 months, 18 months, and 26 months, were confirmed to have ABM (three $S$ pneumoniae, one $N$ meningitidis $W 135$ ). Three children were previously neurologically normal; the other had spastic four limb cerebral palsy. None of these four children had signs of meningism. One child had depressed consciousness after seizure termination; this was thought to be either post-ictal or a side effect of emergency antiepileptic medication. Only one child was irritable before the onset of CSE. In this infant, the anterior fontanelle was bulging. None of the children had a rash. Two children were thought to have had prolonged febrile seizures; one was thought to have an infected ventriculo-peritoneal shunt and in one child ABM was a differential diagnosis. Antibiotics were not thought to be required in one of the children with presumed prolonged febrile seizures, but antibiotic treatment was commenced immediately in the other three. Three children died as a direct result of ABM (see table 1 for summary of clinical details of the children).

\section{DISCUSSION}

Children with CSE and fever are an important subgroup of children with CSE, accounting for half of all incident cases. Studies of CSE with fever may exclude cases with ABM to comply with the definition of febrile convulsions, but that is a retrospective judgement not appropriate to the emergency management of such cases. Although there are clinical guidelines on the emergency management of the child with CSE, ${ }^{11}{ }^{12}$ and the child with a first short seizure with fever, ${ }^{13}$ there remains uncertainty about the role of parenteral antibiotics, the role of lumbar puncture (LP), and the population risk for ABM in children with CSE with fever. By using two sources to identify cases of CSE, and applying capture-recapture analysis to assess completeness of ascertainment, our study has been able to produce a reliable estimate of the number of incident cases of CSE.
Our data suggest that the population risk of ABM, in children with CSE and fever, is higher than would be expected in a population of children who had short seizures with fever $(15-18 \% \vee 1.2 \%)$. Therefore for management purposes, they should be considered as distinctly different patient populations. The recently published guideline for the management of a child with a seizure recommends antibiotic treatment for children who were drowsy before the seizure, have altered consciousness for more than an hour after seizure, or have meningismus. ${ }^{4}$ These classical symptoms and signs of ABM were not present in any of our patients and given the high likelihood of ABM in patients with CSE with fever, the guideline for use of parenteral antibiotics should be extended to include this population of patients. Only two thirds of patients in our study were managed in this manner.

The national guidelines for the management of seizures with fever in the United Kingdom issued in 1991, and the "practice parameter" for first simple febrile seizures of the American Academy of Pediatrics (AAP) published in 1996, both recommend LP to exclude CNS infection in children with short seizures with fever when there are clinical signs of meningitis, and strongly recommend LP in children under 12 months, even in the absence of clinical signs of meningitis. ${ }^{13}{ }^{14}$ Recent publications in Archives have contributed to a further debate on the role of LP in febrile seizures. Riordan and Cant's review on indications for, and contraindications to LP concluded that unless there is a specific contraindication, LP should be performed if meningitis is suspected. ${ }^{15}$ A leading article by Kneen and colleagues, in support of LP, highlighted the possible detriment to patient care through declining use of LP over the past two decades. ${ }^{16}$ On the other hand, Carroll and Brookfield challenged the need for LP. The authors examined the current guidelines and the available evidence for LP following a short seizure with fever and concluded by advocating fewer lumbar punctures for children with short seizures with fever because of the low risk (1.2\%) of ABM, particularly in the absence of signs of meningitis. ${ }^{2}$ However, this recommendation relates to children with short seizures with fever, and such an approach may not be applicable to children with CSE with fever. Through a population based study we have illustrated that ABM is common in children with CSE with fever and may not be clinically apparent. Thus, the controversy discussed above is of lesser importance in this group of patients. We therefore suggest that the most

Table 1 Clinical details of four children with acute bacterial meningitis presenting as convulsive status epilepticus with fever

\begin{tabular}{|c|c|c|c|c|}
\hline Clinical features & Child 1 & Child 2 & Child 3 & Child 4 \\
\hline Age (months) & 3 & 9.5 & 15 & 26 \\
\hline Gender & M & M & $\mathrm{F}$ & $\mathrm{F}$ \\
\hline PMH & Healthy & $\begin{array}{l}\text { Ex-premature } \\
\text { Cerebral palsy } \\
\text { VP shunt in situ } \\
\text { Hydrocephalus }\end{array}$ & Healthy & Healthy \\
\hline Preceding symptoms & Irritable hours before & Intermittent pyrexia $\times 1 / 52$ & Intermittent pyrexia $\times 1 / 7$ & $\mathrm{Nil}$ \\
\hline Temperature & $38.2^{\circ} \mathrm{C}$ & $38.1^{\circ} \mathrm{C}$ & $38.8^{\circ} \mathrm{C}$ & $39.1^{\circ} \mathrm{C}$ \\
\hline \multicolumn{5}{|l|}{ Seizure } \\
\hline Focal features & No & Yes & Yes & No \\
\hline Characteristics & Continuous & Intermittent & Continuous & Continuous \\
\hline Type & Tonic-clonic & Tonic & Tonic-clonic & Tonic-clonic \\
\hline Duration (h) & $0.5-1$ & $>2$ & $1-2$ & $1-2$ \\
\hline Meningism & Absent & Absent & Absent & Absent \\
\hline Rash & $\mathrm{Nil}$ & Nil & $\mathrm{Nil}$ & Nil \\
\hline $\mathrm{WBC}\left(\times 10^{12} / \mathrm{I}\right)$ & 38.4 & 19.1 & 21.3 & 5.1 \\
\hline Neutrophils $\left(\times 10^{12} / \mathrm{l}\right)$ & 28.1 & 12.0 & 17.2 & 1.6 \\
\hline $\mathrm{C}$ reactive protein (mg/l) & 285 & 52 & 279 & 64 \\
\hline Preliminary diagnosis & $A B M$ & Infected VP shunt & PFC & PFC \\
\hline Immediate antibiotics & Yes & Yes & Yes & No \\
\hline Outcome & Severe neurological deficits & Death & Death & Death \\
\hline Organism in CSF & $S$ pneumoniae & $S$ pneumoniae & $S$ pneumoniae & $N$ meningitidis \\
\hline
\end{tabular}




\section{What is already known on this topic}

- The population risk of acute bacterial meningitis ( $A B M)$ in short febrile seizures is low (0.4-1.2\%)

- Investigation for ABM may not be necessary in cases of short febrile seizures unless other evidence of meningitis is present

- Hospital based studies suggest that prolonged febrile seizures (of at least 15 minutes duration) are more likely to be associated with $A B M$ than those with short seizures with fever

- Early treatment of meningitis may decrease morbidity and mortality

appropriate management for children with CSE with fever is to start parenteral antibiotics early, perform an LP when there are no contraindications, and then base duration and type of therapy on CSF findings. Early treatment of ABM may decrease morbidity and mortality. ${ }^{17-19}$ Alternative management strategies have pitfalls as discussed below.

One approach is to withhold antibiotics during a period of observation. ${ }^{420}$ Those children that return to normal within the period of observation may not require antibiotics. However, normal physiological recovery following CSE as well as the side effects of emergency antiepileptic drugs, is variable. One of our cases was thought to be post-ictal, and four hours later developed cerebral herniation. Thus, observation alone may not be appropriate in the child with CSE with fever.

One could modify the above approach by considering the results of blood investigations for evidence of inflammation. However, routine blood tests are not always good discriminators of meningitis, and markers of acute phase response may be absent in the early course of meningococcal disease. $^{321}$ One of our cases had meningococcal meningitis but had a normal white blood cell count, and a mildly raised C reactive protein (see table 1). Thus a "wait for blood test" policy before early antibiotic treatment may have severe and potentially fatal consequences, and is not an appropriate management option.

Another management option, again not to be recommended, would be to do an early LP (in the immediate postictal period) before commencing antibiotics. The potential benefits of early LP include making an early diagnosis of CNS infection, determining appropriate prophylaxis for close contacts, public health monitoring, decreased spending on prolonged therapy and hospitalisation, and facilitating discussions with parents. CSF microscopy will exclude or confirm the diagnosis of meningitis in most cases, as it is rare to isolate a pathogen after normal microscopy on CSF. However, up to $8 \%$ of children with meningococcal meningitis may have a normal CSF, ${ }^{22}$ and Gram stain of CSF will demonstrate an organism in only $68-80 \%$ of samples. ${ }^{23}$ Children with ABM may have further seizures after initial seizure termination, cardiorespiratory compromise, and/or raised intracranial pressure, all of which are contraindications to LP due to the risk of fatal cerebral herniation. ${ }^{15}$ Furthermore, previous studies have suggested that there is a temporal association between LP and herniation. ${ }^{24} 25$ Therefore, the risk of herniation far outweighs the benefit of knowing the pathogen from an early LP, particularly when the pathogen may also be identified through a delayed LP as discussed below.

Immediate administration of antibiotics in the absence of CSF confirmation of ABM may be appropriate, but a marker

\section{What this study adds}

- The population risk of $A B M$ in convulsive status epilepticus (CSE) with fever is much higher than that of short febrile seizures (15-18\% v 0.4-1.2\%)

- The classical symptoms and signs of meningitis may be absent in CSE with fever

- In North London, the emergency management of CSE with fever in a significant proportion of children does not include treatment and investigation for $A B M$

- The most appropriate management for children with CSE with fever is to start parenteral antibiotics early, perform an LP when there are no contraindications, and then base duration and type of therapy on CSF findings

for $\mathrm{ABM}$ is then required to guide duration of therapy. ABM can be confirmed on a delayed LP. The cellular and biochemical changes remain in CSF up to 44-68 hours after the start of antibiotic treatment, and polymerase chain reaction for microbial DNA and RNA is highly sensitive and specific. $^{23}$

In the absence of such a marker, all children with CSE with fever may need to complete a full course of parenteral antibiotic therapy in order to avoid partially treated meningitis. Blood cultures are not an adequate marker of CNS infection. If taken before administration of antibiotics, they are positive in $23 \%$ of cases of meningococcal meningitis without a rash, and $80-90 \%$ of cases of $S$ pneumoniae and $H$ influenzae meningitis. ${ }^{15}$ Even if microbes are isolated in blood samples, there remains no conclusive proof of CNS infection. Clinical improvement may be considered as a marker, but improvement may be due to treatment. Thus, immediate administration of antibiotics should be followed by delayed LP.

This case series suggests that there should be a high index of suspicion for ABM in the child with CSE with fever. Early treatment with parenteral antibiotics and a delayed lumbar puncture when there are no contraindications may be the most appropriate management.

\section{ACKNOWLEDGEMENTS}

We thank all members of the NLSTEPSS collaborative group for their invaluable help in data collection, and all the medical, nursing, and administrative staff who have reported cases of convulsive status epilepticus to NLSTEPSS. We are grateful to Helen Bedford of the Paediatric Epidemiology and Biostatistics Unit, Institute of Child Health, London, for her advice.

\section{Authors' affiliations}

R F M Chin, B G R Neville, R C Scott, Neurosciences Unit, Institute of Child Health, University College London, and Great Ormond Street Hospital for Children NHS Trust, London, UK

Rod Scott is supported by the Wellcome Trust. Research at the Institute of Child Health and Great Ormond Street Hospital for Children NHS Trust benefits from R\&D funding received from the NHS Executive.

\section{REFERENCES}

1 Verity CM, Butler NR, Golding J. Febrile convulsions in a national cohort followed up from birth. I. Prevalence and recurrence in the first five years of life. BMJ (Clin Res Ed) 1985;290:1307-10.

2 Carroll W, Brookfield D. Lumbar puncture following febrile convulsion. Arch Dis Child 2002;87:238-40.

3 Offringa M, Moyer VA. Evidence based paediatrics: evidence

based management of seizures associated with fever. BMJ

2001;323:1111-14. 
4 Armon K, Stephenson T, MacFaul R, et al. An evidence and consensus based guideline for the management of a child after a seizure. Emerg Med $J$ 2003;20: 13-20.

5 Green SM, Rothrock SG, Clem KJ, et al. Can seizures be the sole manifestation of meningitis in febrile children? Pediatrics 1993:92:527-34.

6 Joffe A, McCormick M, DeAngelis C. Which children with febrile seizures need lumbar puncture? A decision analysis approach. Am J Dis Child 1983;137:1153-6.

7 Offringa M, Beishuizen A, Derksen-Lubsen G, et al. Seizures and fever: can we rule out meningitis on clinical grounds alone? Clin Pediatr (Phila) 1992;31:514-22.

8 Brenner $\mathbf{H}$. Use and limitations of the capture-recapture method in disease monitoring with two dependent sources. Epidemiology 1995;6:42-8.

9 Wittes JT, Colton T, Sidel VW. Capture-recapture methods for assessing the completeness of case ascertainment when using multiple information sources. J Chronic Dis 1974;27:25-36.

10 Rahi JS, Dezateaux C. Measuring and interpreting the incidence of congenital ocular anomalies: lessons from a national study of congenital cataract in the UK. Invest Ophthalmol Vis Sci $2001 ; 42: 1444-8$.

11 Appleton R, Choonara I, Martland T, et al. The treatment of convulsive status epilepticus in children. The Status Epilepticus Working Party, Members of the Status Epilepticus Working Party. Arch Dis Child 2000;83:415-19.

12 The Advanced Life Support Group. Advanced Paediatric Life Support. The Practical Approach, 3rd edn. Manchester: ALSG, 2000.

13 American Academy of Pediatrics. Provisional Committee on Quality Improvement, Subcommittee on Febrile Seizures. Practice parameter: the neurodiagnostic evaluation of the child with a first simple febrile seizure. Pediatrics 1996;97:769-72.
14 Joint Working Group of the Research Unit of the Royal College of Physicians and the British Paediatric Association. Guidelines for the management of convulsions with fever. BMJ 1991;303:634-6.

15 Riordan FA, Cant AJ. When to do a lumbar puncture. Arch Dis Child 2002;87:235-7.

16 Kneen R, Solomon T, Appleton R. The role of lumbar puncture in children with suspected central nervous system infection. Arch Dis Child 2002:87:181-3.

17 Welch SB, Nadel S. Treatment of meningococcal infection. Arch Dis Child 2003;88:608-14

18 Beaman MH, Wesselingh SL. 4: Acute community-acquired meningitis and encephalitis. Med J Aust 2002;176:389-96.

19 Aronin SI, Peduzzi P, Quagliarello VJ. Community-acquired bacterial meningitis: risk stratification for adverse clinical outcome and effect of antibiotic timing. Ann Intern Med 1998;129:862-9.

20 Brogan PA, Raffles A. The management of fever and petechiae: making sense of rash decisions. Arch Dis Child 2000:83:506-7.

21 Pollard AJ, Britto J, Nadel S, et al. Emergency management of meningococcal disease. Arch Dis Child 1999;80:290-6.

22 Wylie PA, Stevens D, Drake W, et al. Epidemiology and clinical management of meningococcal disease in west Gloucestershire: retrospective, population based study. BMJ 1997;315:774-9.

23 Feigin R, Pearlman E, Feigin R, et al. Bacterial meningitis beyond the neonatal period, 4th edn. Philadelphia: WB Saunders, 1998.

24 Dodge P, Swartz M. Bacterial meningitis - a review of selected aspects II. N Engl J Med 1965;272:954-60.

25 Rennick G, Shann F, de Campo J. Cerebral herniation during bacterial meningitis in children. BMJ 1993;306:953-5.

\section{IMAGES IN PAEDIATRICS}

\section{Monoarthritis; remember to ask the child}

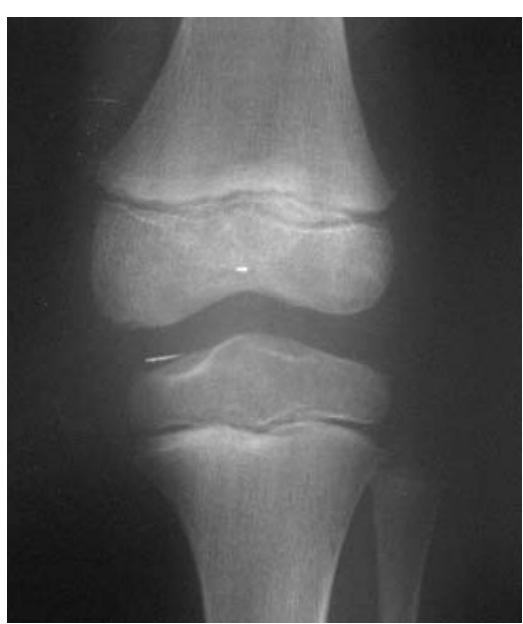

Figure 1 Staples in the knee joint. n 8 year old boy was referred by his general practitioner to the paediatric outpatient department with pain and swelling of his left knee. This had been present for three months, during which the boy had attended the general practitioner on more than one occasion. A presumptive diagnosis of reactive arthritis had been made initially and he was treated with ibuprofen. His mother had a definitive diagnosis of rheumatoid arthritis and was concerned that he might be developing this.

When seen in outpatients, it was clear that the knee had been swollen for around six months. The swelling was more or less continuous. The pain was variable. The knee had been particularly painful at the onset. Intermittent stiffness of the knee was also present as was an intermittent limp.

On examination the child looked very well. His left knee had a small effusion and was slightly warmer to touch than the right but was not particularly tender. Movements were normal apart from minor limitation of extension.

When the child was asked if he had any idea why his knee was sore, he stated with some conviction that he knew what had caused it. He was in the school playground one day and knelt down to tie his shoelace, whereupon a sharp metal object, which he referred to as a staple, penetrated his knee. He had told this to his mother who dismissed it as a cause of his difficulties as she had had a look at his knee and could not see any obvious sign of penetrating injury.

$x$ Ray examinations of the knee showed two radio-opaque foreign bodies. The boy was referred to the orthopaedic surgeons for arthroscopy and further management. Subsequently he had the staples removed.

B S Rayen, A Chapman Dumfries \& Galloway Royal Infirmary, UK

Correspondence to: Dr B S Rayen, Department of Paediatrics, Dumfries \& Galloway Royal Infirmary, Bankend Road, Dumfries DG1 4AP, UK; drrayen@yahoo.com 